\title{
Thermoelastic solution of a functionally graded variable thickness rotating disk with bending based on the first-order shear deformation theory.
}

\begin{abstract}
A theoretical solution for thermoelastic analysis of functionally graded (FG) rotating disk with variable thickness based on first-order shear deformation theory (FSDT) is presented. Material properties and disk thickness profile are assumed to be represented by power law distributions. A semi analytical solution for displacement field is given under two types of boundary conditions applied for solid and annular disks. The effects of the material grading index and the geometry of the disk on the stress and displacement fields are investigated. Mechanical responses homogeneous disks versus FG disks are compared and verified with the known results in the literature. It is seen that the transverse displacements in FG solid disks with roller support condition at the outer surface remain between the minimum displacement value for the full-ceramic disk and the maximum displacement value for the full-metal disk. It is found that the transverse displacements in FG mounted disks with free condition at outer surface may not lie in between the displacement values for full-metal and full-ceramic disks. It is observed that the absolute moment resultant for FG mounted disk with concave profile is lowest compared to the FG mounted disk with linear or convex profile. It can be concluded that the gradation of the metal-ceramic components and the geometry of the disk are significant parameters in the thermomechanical responses of FG disks.
\end{abstract}

Keyword: Functionally graded materials; Rotating disk; Shear deformation; Thermoelastic; Variable thickness. 